\title{
The Structure of the Atom-II
}

\author{
The Physical and Chemical Properties of the Atom Explained in the Light of Modern Theory and Experiment
}

By Sir J. J. 'Thomson, F.R.S.

\section{Continued from Scientific American Supplement No. 1944, page 219, April 5, 1913}

ON the last occasion I explained various processes by which it is possible to extract from atoms, whether belonging to a gas or to a solid, small particles which are negatively electrified. No matter what the source may be from which these particles are derived, they are absolutely identical, each carrying the same charge and possessing the same mass. These negatively and possessing the same mass. These negatively whatever is left must necessarily be positively electrified, since it is impossible to generate electricity of the one kind without simultaneously liberating that of the other kind. An interesting question thus arises as to what is the nature of the positively electrified bodies left behind after the abstraction of the negative corpuscle. It has been shown that the latter are far smaller than any known atom, and it is conceivable that the positively electrified residue is also smaller than the atom. A few experiments suffice, however, than the atom. A few experiments suffice, however, to show that there is a great difference between the
carriers of positive electricity and the negative corpuscles.

[This difference the speaker illustrated by means of a glass
vssel consisting of two large bulbs united by a wide tube. vessel consisting of two large bulbs united by a wide tube.
In the latter was placed a perforated cathode. On turning In the latter was placed a perforated cathode. On turning
on the current it was scen that the negative carriers above on the current it was scen that the negative carriers above
the cathode gave out a bluish-green glow, and where they struck the glass, produced a greenish phosphorescence. suffice to twist this negative discharge in any direction. Th suffice to twist this negative discharge in any direction. The
positive discharge, on the other hand, which was attracted positive discharge, on the other hand, which was attracted
down toward the perforated cathode and passed through it
into the into the lower bulb, glowed with a reddish light, and where
it impinged produced a bright red phosphorescence. A small it impinged produced a bright red phosphorescence. A small
magnet held ncar this lower bulb had anparently no effect magnet held ncar this lower bulb had anparently no effect
whatever on the discharge. This showed, the lecturer stated. whatever on the discharge. This showed, the lecturer stated.
that the negative carricr was quite different in character from the positic one. In another experiment Prof. Thomson causcd a negative discharge to impinge upon some lithium chloride. The latter then phosphoresced with an intense stecly blue color where struck, and it was stated that the spectrum was a continuous one. Reversing the direction of the current, a corresponding stream of positively electrifled particles was directed onto the lithium chloride. The
phosphorescence was then red, and this. it was stated, gave phosphorescence was then red, and this. it
a line spectrum showing the lithium lines.]

Experiments of this kind suffice to show that there is a very great difference between the negative and the positive carriers. Though the latter cannot be sensibly deflected by a small magnet. more intense fields give a measurable deflection, and by simultaneously subjecting this positive discharge to powerful magsubjecting this positive discharge to powerful mag-
netic and electric fields, it is possible to deduce, from the deflection produced by the joint action of the two, the deflection produced by the joint action of the two,
the mass of the positive particles. When this is done, no trace is found of any mass smaller than those of known atoms. the weights found coinciding, in fact, with the masses of the atoms and molecules which one would expect to find in the tube. The positive particles, therefore, are charged atoms and molecules, while the negative carriers are charged corpuscles, or, rather, corpuscles simply, since a corpuscle was essenrather, corpuscles simply, since a corpuscle was essen-
tially a negative charge. These corpuscles, moreover, tially a negative charge. These corpuscles, moreover, are all identical, while there are as many different
kinds of positive carriers as there are different kinds of atoms and molecules in the tube. This is well shown in Fig. 1, reproduced from a photograph obtained by bombarding a plate with positive carriers, after the latter have traversed a combined magnetic and electric field. Each curve on the plate corresponds to particles of a particular kind. The least deflected are the heaviest, the outermost curve corresponding to the atom of hydrogen, and the innermost to that of mercury; and there are, it will be seen, a number of mercury; and there are, it will be seen, a number
of curves on the plate. With the negative corpuscles, on the other hand, the photograph would consist of a single curve.

Curves, such as those in Fig. 1, give quite a lot of information about atoms, the mass of each of which can be determined by measuring the curves. Some of the curves are produced by charged atoms, while others turn out to be due to charged molecules, so that others turn out to be due to charged molecules, so that
the photograph shows that atoms exist as well as molethe photograph shows that atoms exist as well as mole-
cules. With helium, however. only one curve is obtained, thus showing this gas to be monatomic. By using a sufficiently fine pencil of rays very sharp curves can be obtained, which afford definite proof that the atoms of any element have all exactly the same mass. If they had not, the curve obtained would be a blurred riblosn rather than a sharp line.

* 'The second of a course of lectures dellvered at the Royal Institution, London, and reported in Engineering.
The fact that the positive particles have the same mass as the atoms and molecules in the tube shows the tearing out from an atom of one of the negative corpuscles, and that such corpuscles form accordingly constituents of every atom. It is important to know how many of these corpuscles there are in an atom. The facts already recited show that at least one can be torn out, but by studying the curves in the photograph it has been proved that from some atoms many more corpuscles can be removed. The carbon atom is found carrying both one charge and two charges of positive electricity, and at least two corpuscles can also be removed from the atom of oxygen, while from that of mercury as many as eight can be taken, and from krypton any number up to four or five. Heavy from krypton any number up to four or five. Heavy corpuscles, but the lighter atoms lost fewer, and from $\mathrm{H}$ I have not, so far, succeeded in abstracting mor than one, this element being peculiarly economical in its expenditure of corpuscles. From oxygen and carbon not more than two corpuscles have been removed. In a later lecture I shall, however, bring forward reasons for believing that the number of corpuscles
weight.

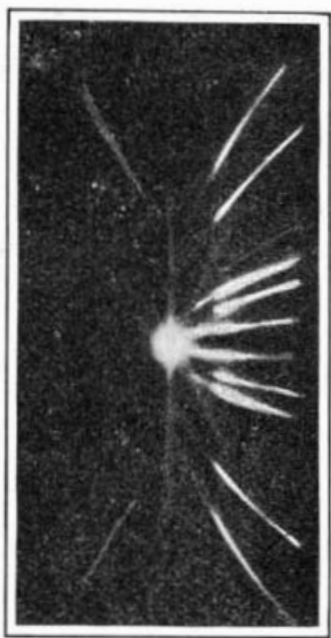

Fig. 1

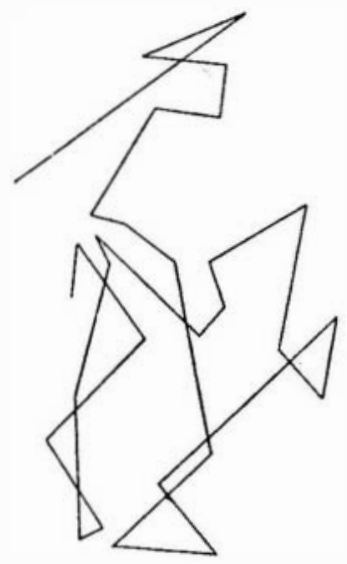

Fig. 2.
Referring again to Fig. 1, it will be seen that in addition to the curves on the right, corresponding to positively charged atoms and molecules, there are also traces, on the left, of curves due to similar bodies carrying negative charges. It follows, therefore, that the atoms can not merely give up a corpuscle, but they are also capable of pleking up and holding on to these negative particles.

The process by which they acquire this negative harge is as follows:

The positively charged particle, after passing the opening in the cathode, enters a space in which the gases present have been broken up by the stream of particles, which ionizes the gas through which they pass, liberating negatively and positively charged
bodies. The rapidly moving positive particle, after bodies. The rapidly moving positive particle, after
passing through the cathode, attracts one of the negapassing through the cathode, attracts one of the nega-
tive charges thus liberated, and becomes, accordingly, for the moment neutral. Not content with this, however, it attracts an additional negative charge, and thus gets deflected by the magnetic and electric fields to the left-hand side of Fig. 1 .

Its affinity for this additional negative charge must be very considerable, as it is moving at the prodigious rate of about 50,000,000 centimeters per second, and rate of about $50,000,000$ centimeters per second, and
has to pick up the negative charge as it rushes past. has to pick up the negative charge as it rushes past.
The attraction for this negative charge must therefore be very strong. It has been found that not all the gases, represented on the right-hand side of the photograph, are also represented on the negative side. This turns out to be very largely a matter of chemical constitution. Of all gases oxygen most readily picks up this additional negative charge (see illustration on page 269 of SuPPLEMENT No. 1921) and chlorine, another electro-negative gas, acts similarly. There are, however, other bodies, not usually classed as electroever, other bodies, not usually classed as electro-
negative, which are nevertheless represented on the negative, which are neverth
left-hand side of the figure.

Hydrogen is one example and carbon another. In this connection it is of interest to note that if the carbon compound in the tube is "unsaturated," that carbon atom, as in acetylene and ethylene, it is found represented on the negative side of the photograph, represented on the negative side of the photograph,
being then capable of picking up an additional negative being then capable of picking up an additional negative
charge. With saturated compounds, however, such charge. With saturated compounds, however, such
as marsh gas, carbon is found only on the positive side as marsh gas, carbo
of the photograph.

The photographs may also be used to determine if any elements not yet known are present in the tubes. At Cambridge we have thus found lines corresponding to a number of gases not yet recognized. The one of most interest has an atomic weight of 3 . This line is very marked in a photograph taken when the gases driven out of platinum by bombarding it with the eathode rays form the subject of the experiment. It was found that, even after three days' bombardment, traces of this line could still be detected. Other metals, indeed, have been bombarded for a week without getting rid of this gas.

I shall next consider various ways of arriving at the size of the atom. This must obviously be very small, but both its weight and its size are now known accurately within about one per cent. One of the simplest methods in conception, though not in execution, is due to Perrin. The kinetic theory of gases shows that every particle present in a gas, at a stated temperature, contributes the same amount to the total pressure observed. Thus if $N$ are the total number of molecules of a gas present in a given volume, then the total pressure exerted is equal to $N a$, where $a$ depends only on the temperature of the gas. In other words, $N$ being the number of particles, $a$ is the average contribution of each to the pressure. It follows from the kinetic theory that $a$ is the same whatever the nature of the particle in question, whether it is hydrogen, oxygen, mercury, or the heaviest that could be found. It will be evident therefore that if $a$ were known, and also the pressure, the value of $N$ could be found, this being the number of molecules in the mass under consideration.

How, then, ean $a$ be found? If the particles were so big that $N$ could be counted, we could, by measuring the pressure, find $a$, which, as stated, is the same for all molecules. This, though somewhat crudely expressed, is practically what Perrin does. The first step necessary is to find something which, while constituted of comparatively big particles, behaves nevertheless like a gas, since the molecules of actual gases are far too minute to be visible even microscopically. It has long been known that small particles suspended in a liquid are in a continuous state of agitation, the so-called Brownian movement. They dart hither and thither, being never at rest, but change their direction of motion abruptly, just as the molecules must do in a gas. Prof. Perrin has actually mapped out the paths followed by such a particle when observed for a small time, and this is reproduced in Fig. 2. It had early been suggested that the Brownian movements were equivalent to the molecular movements indicated by the kinetic theory. The small suspended particles behaved, in fact, in just the normal way that the molecule of a gas would do. This fact seemed to promise the possibility of getting something equivalent to gas with "molecules" big enough to observe and count, and this forms, so to speak, an artificial "gas," in which $N$ could be counted and the corresponding pressure measured. The molecules of a gas are. however, al of the same size. Hence it was necessary to obtain a very fine powder as nearly uniform as possible to represent them. The most elaborate of Perrin's experiments were made with two powders, one being gamboge suspended in water, and the other mastic dissolved in alcohol and dropped into water. The powders thus obtained were not originally uniform, the particles being of different sizes, and as they stood they were quite unsuitable for the experiment contemplated. By laboriously "centrifugating" them for a period o over three weeks, Prof. Perrin finally obtained powders of extreme uniformity and great fineness. Assuming
that these particles would behave like the molecules of a gas, he then proceeded to find $N$ by actual count and to determine also the corresponding pressure. The particles were too fine to be directly visible in the microscope, but on submitting the liquid in which they were suspended to a very strong illumination. they appeared as bright specks, something like the particles of dust visible in a strong beam of light passing 
through the air of an average room. By counting these specks it was possible to determine $N$, but the question next arose as to how the corresponding pressure was to be determined. This pressure was not that of the atmosphere nor anything like it.*

Everyone knows that the density of the air diminishes with the height above sea-level, the difference being equal to the weight of the intervening air. The same in a long vertical column it is found when the steady state is attained, that the density of the specks is much state is attained, that the density of the specks is much
greater below than higher up. At the bottom the specks are crowded together, while at the top they are quite widely separated. At every layer of the column the pressure is equal to $N a, N$ being the number in that layer. If a count is taken at two different
levels, the number at one level is, say, $\mathrm{N}_{1}$, and at the

* [On this point the following may be of assistance to some of our readers: If a gas be cnclosed in a vessel it
cxerts a certain pressure on the sides. This pressure is due cxerts a certain pressure on the sides. This pressure is due
simply and solely to the impact of the molecules, which are darting hither and thither in a random fashion similar to that represented in Fig. 2 supra. Since none of Prof. Perrin's particles could escape from the surface of the liquid, his
column of water formed in effect a closed vessel, which confined his artificial gas. The particles constituting the latt being in continuous motion were continuously coming into contact with the sides of the tube, and it is the pressure pro
duced by their impact which corresponds to the pressurc o duced by their impact which corresponds to the pressure o
the "gas" and which it was necessary to measure.-The Edithe "gas" and which
tor of Engineering.] other $N_{2}$. The pressure difference is therefore $\left(N_{1}-N_{2}\right) a_{2}$ and this must be equal to the weight of all the particle between the two levels. If $r$ is the radius of a particle its volume is $\frac{4 \pi r^{3}}{3}$, and its weight in the suspending fluid is equal to $\frac{4 \pi r^{3}}{3} \times g(\rho-\sigma)$, where $\rho$ is the density of the particle and $\sigma$ the density of the fluid. Hence, if $N$ is the mean number in unit height, the weigh in a thickness equal to $t$ is

$$
N-\frac{4}{3} \pi r^{3} g t(\rho-\sigma) \text {. }
$$

Hence we finally have the relation

$$
a\left(N_{1}-N_{2}\right)=\mathrm{N} \frac{4}{3} \pi r^{3} g t(j-\sigma) .
$$

Hence $a$ can be found by counting $N$ at different levels, nd measuring the radius and density of the particles. The method counting $N$ which would naturally occur to an experimenter would be to take photoever, that this plan would not work, the plates being never sufficiently distinct. Had it been practicable to work in the way suggested, many months' labo would have been saved. Actually Prof. Perrin counted them, so to speak, "by hand," sitting at the microscope and making a record whenever he saw the flash of light, due to one of the specks crossing the field. By taking hundreds of observations he thus got by actual count a kind of average value of $N$ at a number of different layers.

The next quantity to be determined was $a$, the radius of the particles. This is extremely small, being far too minute for measurement by the micrometer in the microscope. The measurement, therefore, was effected indirectly by observing the rate at which the particles subsided through liquids such as water. As explained in the first lecture, this rate depends on the size of the particle, and by measuring it the radius of the particles can be deduced by means of Stokes's law. The particles used by Perrin subsided extremely slowly; but by taking care that their motion was not disturbed but by taking care that their motion was not disturbed
by convection currents, the rate of fall can be found with great accuracy. This was one of the methods used by Perrin to determine the size of his particles.

He had next to justify his assumption that the particles in his emulsions behaved like the molecules of a gas. In the case of a gas $a$ is the same whatever the gas might be; and Perrin, in repeating his experiments with different powders, found that in this case also $a$ was the same whatever the powder used, getting identical results with gamboge and with mastic. Another check was obtained by showing that $a$ was the same whatever the liquid used in forming the emulsion, and by these two searching checks he justified his assumpgas.

(To be continued.)

\title{
The Dangers of Street Traffic and Danger Signals
}

\author{
An Educational Campaign
}

By Frederick Remsen Hutton, M.E., Sc.D.

The American Museum of Safety in New York has been conducting a campaign of education among the school children, to bring home to them the common dangers of the home and their play-places. The gasstove and the opened knife, at home; the trolley, the live wire, and the fast-moving or heavily-laden vehicle live wire, and the fast-moving or heavily-laden vehicle
where they play, have all heir potential or present dangers to the child whose mind is on something of vastly greater interest for the moment. A semi-automatic sense of danger must be awakened in the city child, like an eighth sense, to turn his head in the direction from which danger is approaching; and on the other hand, traffic must follow defined paths, and use standard warning procedure.

This campaign is being backed and promoted by the Rapid Transit interests of Brooklyn, for they havı stake in making the streets safe both from a humanitarian and a business point of view; and the printing of leaflets and the provision of school lectures are th methods in use.

The eity street is the playground of many children, both from necessity and from choice. It is the only open place under the sky in many parts, and it is the social center and the gathering place for playmates and for partners in the group games. The quick-moving, electric trolley car, with its relatively silent approach, has yet the safety attaching to a fixed path or track The child ean early learn to keep off the rails and the right of way, and when it does trespass, to look out fo "the cars." But the free-moving vehicle has the use of the rest of the roadway in common with the pedestrian and the playing child. Both child and adult "on their lawful occasions" are there, and they must avoid meeting each other. What standards shall be established ing each other.

There are some absurd proposals that all individual vehicular traffic shall be stopped at intervals; this would defeat its own purpose, and be hardly enforce able, except in spots, and the whole subject is too broad to be discussed in its entirety in a short article. But there are certain standards which seem so possible for the user of the wheeled vehicle that it may be worth while to discuss them.

(1) The Controlled Traffic.-If all vehicles head on way, and only turn around at the corners, or street intersections, then all on foot need only look for danger from one direction. On the right of the center, you look over the left shoulder; on the left of the center, you look for danger from the right hand. No child should ever play in the intersections of streets, and no pedestrian or vehicle should ever "cut the corners."

(2) The Classified Traffic.-Since it is the Rule of the Road that the faster vehicle passes the slower on the Road that the faster vehicle passes the slower on
the right of the former and by turning to the left hand the right of the former and by turning to the left hand
of the slower, the faster vehicles will always be in the center of the highway, or farther away from the sidewalks of the street. This is the first classification, and rightly makes the center of the street the more dangerous part. But a second classification is that of the ordinary and the preferred class of vehicle as
respects the right of way. The hospital ambulance and the fire-service vehicles on their errands of lifesaving or rescue of lives or property, are entitled to speed, and the center of the street. Pedestrians should get on the sidewalks, and vehicles of the lower classes should go to the right of the highway and stop there No child should be or remain on the roadway when a fire or hospital vehicle is exercising its paramount right of way.

(3) The Standard Type of Warning Signal.-No vehicle should have or sound the warning signal of a superior elass. The powerful gong rapidly sounded is the accepted signal for the ambulance in this part is the accepted signal for the ambulance in this part
of the world, and the high-pitched steam whistle or of the world, and the high-pitched steam whistle or
siren or the swinging bell is the fire signal. For others to use the emergency standards is to lower the warning value of the real ones, and should be forbidden by ordinance. No man should cry "Wolf" like the shepherd in the fable, to get a right of way to which he is not entitled, else he breeds carelessness in the face of real need.

(1) A Standard Quality of Warning Signal.-A warning signal must not only impress sound waves on the drum of the ear, but it must reach the mind behind the ear, and cause volitional action. The action should be partly automatic, in the sense that action should follow the sense-impression quickly enough to eseape accident, if it comes near. Hence it will be of the greatest value if the mind can be trained to recognize the signal of the fast-moving vehicle, and at the same time to judge the distance from which the sound proceeds. Most drivers are watching the roadway about 200 feet in front of the vehicle on the city streets, or 200 feet in front of the vehicle on the city streets, or
at about one block away. He probably sounds his sigat about one block away. He probably sounds his sig-
nal for the person on the street at about this same distance. Hence the signal should carry its alarm notice over that distance to even a deaf or a slowmoving person and against the wind and other noises of the street. It should impress the mind with the notion of the speed necessary to avoid impact. The warning signal should be of a quality not attaching to any other street noise; and it should be audible to any other street noise; and it should be audible not jump with a nervous movement to avoid something which seems at his side before he knew that it was anywhere near him.

(5) The Warning Signal Should Not be Sounded Unnecessarily.-To do so is not only to make the street of disregarding the signal when it is full of real meaning. A noisy street is dangerous when the danger signals cannot be heard; the quieter the street the more easily is the attention caught.

(6) A true musical note (one with a definite number of air-waves per second) is not as serviceable for arousing attention and for warning as a pure noise, unless so-called siren is a pure tone at all points of its range, but to make it carry sudden warning, it has to be so loud that it is rightly to be forbidden where noise is an objection. The short explosive note of types of signal, where a steel diaphragm is set vibrating by an electric motor on the principle of Savart, makes
the first sound waves as effective as those which leave the signal later, and this is its best claim to be a safety device, in the sense that the American Museum of Safety uses that term. The common pneumatic reed actuated from a hand-bulb cannot be heard in noisy traffic or against strong winds for any considerable distance, or even a city block, and is scarcely, therefore, entitled to be classed among safety devices, in the Museum sense.

While it is safe to say that a standardization along the above lines will reduce the number of collisions on the streets, there still remains the irreducible minimum whose origins are carelessness and absence of mind.

\section{Liquid Fuel ${ }^{*}$}

The Cantor lectures, just concluded by Prof. Vivian B. Lewes on "Liquid Fuel," provide exactly the kind of information required at this moment to steady public opinion and to indicate the probable course of development of a vast industry. So far as Great Britain is concerned, the wider the fields of distribution are separated, the better it will be for naval, mercantile, and industrial purposes, and it is to be hoped that the sketch presented by Prof. Lewes, representing the extent and value of the fields thus far opened, will induce young engineers and geologists to take up the task of prospecting, surveying, and operating in new areas. There is need for discrimination in cegard to the products of the respective oil fields so far discovered and worked, for it must not be assumed that they demand the same initial treatment in each instance. The crude oil of Burmah differs, for example, from that of Rumania, and both these products differ from that of Mexico. Hence, there is ample opportunity for young chemists to apply their knowledge and to seek their fortunes in some of the most attractive countries of the world. The quantities so far being won are impressively great, and the statement that in 1912 the total weight of crude oil taken from the oil fields was between forty and fifty million tons may to some minds suggest early finality; yet the experience of the world's coal fields, and of the unexpected discoveries that are made from time to time, affords ground for hope that such misgivings may be unwarranted. In Mexico, Peru, and elsewhere a considerable proportion of the available oil is used locally, and there is no doubt that the consequences of the improved supplies of fuel thus rendered available in such countries will be a cardinal factor in their future development. It is by optimists rather than by pessimists that success in the problem of the liquid fuel industry will be achieved.

* Reproduced from the Engineering supplement of the london 